\title{
Construction and Testing of a Dual Faraday Cup for Low Vacuum and Environmental Scanning Electron Microscope
}

\author{
S.A. Wight and A.R. Konicek
}

Surface and Microanalysis Science Division, National Institute of Standards and Technology, 100 Bureau Drive, Stop 8371, Gaithersburg, MD 208991-8371

Current measurement is complicated in the low vacuum environment of any of the scanning electron microscopes where the specimen chamber contains a partial pressure of a gas. The primary electrons can be scattered out of the primary electron beam and form a "skirt" that can extend up to hundreds of micrometers away from the primary electron beam impact. This is a function of experimental conditions such as: accelerating voltage, gas composition, gas pressure and the distance the beam travels through the gas [1]. This work was motivated by the efforts of Rattenberger and coworkers to come up with a method to calculate a beam gas path length and total scattering cross section [2]. The goal was to build a dual Faraday cup that would be capable of simultaneously measuring both the unscattered fraction of the primary electron beam and the fraction that was scattered up to $10 \mathrm{~mm}$ from the primary electron beam.

The core of this dual Faraday cup is the $12 \mathrm{~mm}$ diameter by $10 \mathrm{~mm}$ high graphite cylinder. Graphite was chosen for it good electrical conductivity and most importantly its low backscatter yield. It is undesirable to backscatter electrons back out of the Faraday cup. Further to this end the $1 \mathrm{~mm}$ diameter hole was drilled in the top surface at roughly a 10 degree angle to surface normal such that the bottom of the hole is not aligned with the top of the hole. The center conductor of a shielded BNC cable was connected with carbon paint to a hole matching the diameter of the conductor in the side of the graphite. The graphite block with the exception of the top surface was embedded in 25 $\mathrm{mm}$ diameter epoxy for electrical isolation (FIG. 1). A 200 micrometer diameter aperture with an outside diameter of $6 \mathrm{~mm}$ was positioned over the center of the $1 \mathrm{~mm}$ hole in the graphite. It was held in place by drops of carbon paint. The top surface of the epoxy/graphite/aperture was covered with two strips of double stick tape. The tape serves two functions; it is an electrical insulator and it will hold the metal foil in place in the next step. Under an inspection scope an $0.5 \mathrm{~mm}$ by $0.5 \mathrm{~mm}$ square of tape is cut away to reveal the 200 micrometer aperture. A piece of 8 micrometer thick nickel foil is cut to approximately $20 \mathrm{~mm}$ diameter circle with a $3 \mathrm{~mm}$ tab on one side to make electrical contact. A $1 \mathrm{~mm}$ by $1 \mathrm{~mm}$ hole is cut with a scalpel in the center of the nickel foil. Under the inspection scope the foil is placed on the tape such that the 200 micrometer aperture is in the center of the opening. Using a light microscope with a cross-hair reticule, the 25 micrometer platinum aperture is aligned with the 200 micrometer aperture and them held in place with drops of carbon paint. The center conductor of a second shielded cable is silver painted to the tab on the nickel foil. A measurement set collected with the dual Faraday cup $(20 \mathrm{kV}$, spot 3, 30 micrometer projection aperture, water vapor) in the FEI Quanta FEG can be found in FIG. 2.

References

[1] S.A. Wight, Scanning. 23 (2001) 320.

[2] J. Rattenberger, et al., Scanning. 31 (2009) 107. 
[3] Certain commercial equipment, instruments, or materials are identified in this report to specify adequately the experimental procedure. Such identification does not imply recommendation or endorsement by the National Institute of Standards and Technology, nor does it imply that the materials or equipment identified are necessarily the best available for the purpose.

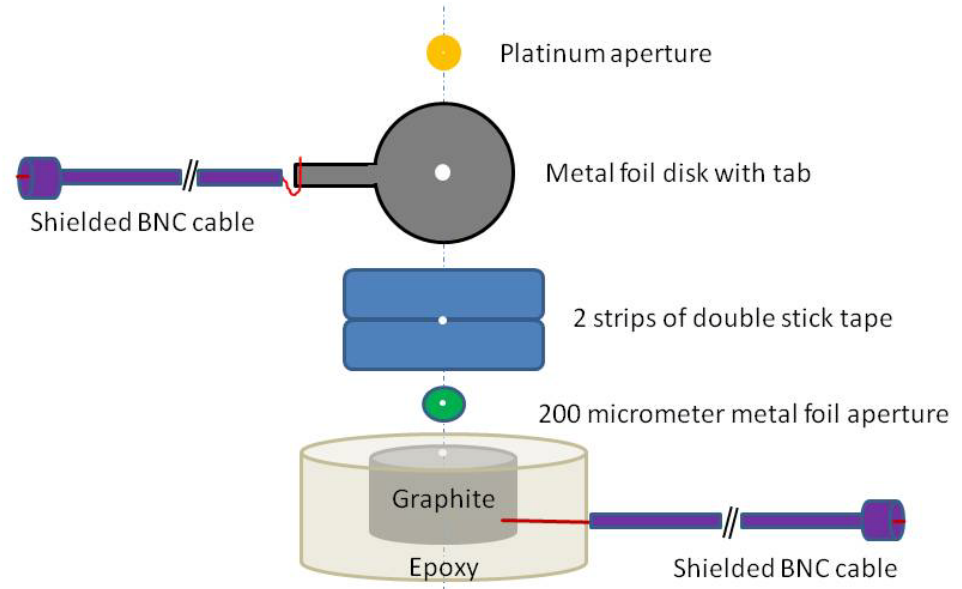

FIG. 1. This diagram presents an exploded view diagram of the construction of the dual Faraday cup. The graphite block with a hole in the center is embedded in epoxy except for the top surface. The center conductor of the shielded cable is connected to the graphite block. A 200 micron metal foil aperture is carbon painted over the hole in the top of the graphite. Two strips of double stick tape with a hole cut out over top of the 200 micron aperture opening. A metal foil disk with a hole to align with the 200 micron aperture is attached to the tape. The center conductor of a second shielded cable is connected to the metal foil. Finally the platinum aperture is aligned over the center of the 200 micron aperture and then carbon painted in place.

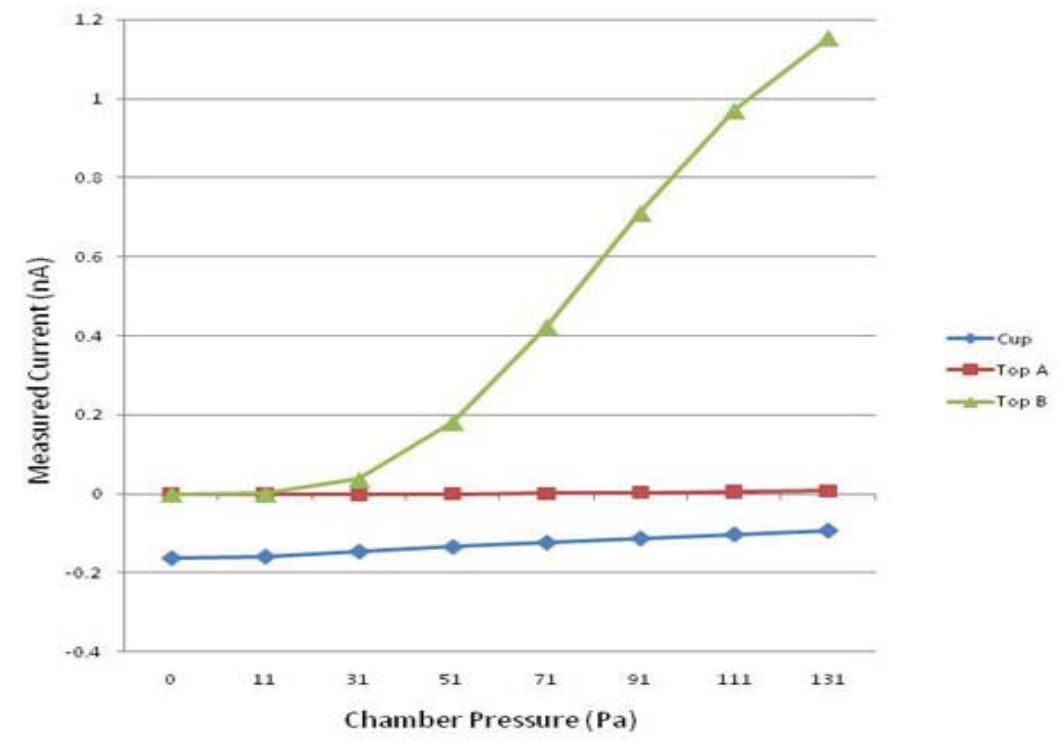

FIG. 2. Current measurement in the buried cup (blue circles) is plotted with current measurements from the top surface when the large field detector is turned off (Top A - red squares) and turned on (Top B - green triangles). Even with the detector off the values go positive at higher gas pressures. 